Voix et Images

voixetimages

\title{
D’où vient Voix et Images?
}

\section{Jacques Allard}

Volume 12, numéro 2 (35), hiver 1987

Jacques Brault

URI : https://id.erudit.org/iderudit/200638ar

DOI : https://doi.org/10.7202/200638ar

Aller au sommaire du numéro

\section{Éditeur(s)}

Université du Québec à Montréal

\section{ISSN}

0318-9201 (imprimé)

1705-933X (numérique)

Découvrir la revue

Citer cet article

Allard, J. (1987). D’où vient Voix et Images? Voix et Images, 12(2), 294-303.

https://doi.org/10.7202/200638ar d'utilisation que vous pouvez consulter en ligne.

https://apropos.erudit.org/fr/usagers/politique-dutilisation/ 


\section{D'où vient Voix et images?}

\section{par Jacques Allard, Université du Québec à Montréal}

J'aimerais dire en toute simplicité d'où vient la revue Voix et images. Rappeler quelques moments de son émergence et certaines conditions de son évolution. Aller rapidement du milieu de nos années soixante jusque vers 1975. De l'Université de Montréal à. l'Université du Québec à Montréal en passant par le Collège Sainte-Marie. D'un groupe à l'autre que constituent souvent les mêmes personnes, tout à la fois enseignants et étudiants, tous à l'école de la révolution tranquille, buissonniers du pays imaginaire.

\section{Deux scènes}

À l'origine, deux scènes. La première est située sur le flanc nord du Mont-Royal où se dresse le monument "modern style" de l'Université de Montréal. L' «alma mater». Là, des étudiants sont regroupés autour d'une table vernie, pour le séminaire bi-mensuel consacré à la littérature canadienne-française. Dans leur jeune vingtaine ou même trentaine, ils se consacrent à leurs études supérieures, tout en enseignant dans divers collèges montréalais ou même, déjà, en cette même université.

ll y a là André Brochu, Ulric Aylwin. Renald Bérubé, Roland Arpin et plusieurs autres dont je suis. Assez souvent se joignent à nous Fernande Saint-Martin et Andrée Yanacopoulo. Au bout de la table, se découpant dans la fenêtre, le maître: assis bien droit, une pipe sceptique entre des lèvres épaisses. Bientôt, son front bombé se plisse sous un panache de fumée odorante. Et. de sa main droite, il retire la bouffarde de sa bouche, repousse ses lunettes à la racine du nez, tout contre des yeux ironiques, et pointe un tuyau fumant vers l'un de nous:

- Et vous, qu'en pensez-vous? dit Albert Le Grand. 
C'est Brochu qui doit répondre à la critique qui vient d'être faite à son exposé sur Bonheur d'occasion. Ou est-ce moi à propos de la Petite poule d'eau? Ou Arpin sur l'Incubation? Ou mieux: Aylwin sur les Chambres de bois? Car celui qu'on appellera bientôt familièrement "Albert» a encore repris sa problématique de l'aliénation et est revenu à son terrain textuel préféré (après l'œuvre de Gide), celui d'Anne Hébert.

Nous sommes tout à la fois ravis et agacés. En cette fin d'après-midi, se redéploient, emmêlées, nos lectures thématiciennes (à la Jean-Pierre Richard, à la Georges Poulet) ou symboliques (façon Ernest Gagnon, s.j.), ou stylistiques (à la Bally revu par Bernard Dupriez) ou sociologiques (inspirées par Jean-Charles Falardeau)... Nous discourons sans complexe sur cette littérature que nous nommons "québécoise" à l'encontre de la plupart de nos professeurs qui parlent toujours de la "canadienne». Nous discourons en toute rigueur françaișe (d'après les bonnes règles de la dissertation enseignées par Bernard Beugnot). Et, dans ce département d'Études françaises, nous militons pour la "québécité». Comme dans la tour phallique où s'affaire, depuis 1964 , Réginald Hamel en son Centre de documentation des lettres canadiennesfrançaises. À la revue Parti pris où nous écrivons (Brochu, Hamel et moi). Nous sommes dans la mouvance nouvelle, socialo-indépendantiste, qui ébranle l'idéologie "canadienne». Et l'année littéraire est fastueuse: paraissent alors tout crépitants, le Couteau sur la table (Godbout), Prochain épisode (Aquin), l'Incubation (Bessette), Une saison dans la vie d'Emmanuel (Blais), l'Âge de la parole (Giguère), l'Afficheur hurle (Chamberland), l'Avalée des avalés (Ducharme). Comment ne pas écrire?

Oủ publier nos analyses littéraires? Parti pris est d'abord politique, et il faut y justifier ce type de travail (voir les articles qu'y publie Brochu). Liberté est un "club" quoi qu'en disent ses théoriciens du "déclubbage» (contre les clubs privés de chasse et de pêche du Québec...); la revue n'a pas, de toute manière, une vocation universitaire. Livres et auteurs canadiens (québécois à partir de 1969) est d'abord une revue de comptes rendus. Sur le Mont-Royal, Études françaises que vient de lancer René de Chantal a beau publier l'étude de Brochu sur Angẻline de Montbrun, sa porte n'en nous parait pas moins étroite que celle de ce département où nous étudions. On y prend notre littérature avec beaucoup de pincettes. Quant aux Archives des lettres de Wyczynski, elles appartiennent au circuit outaouais. Restent des ressources marginales comme l'hebdomadaire Quartier latin, les Cahiers de l'AGEUM, Lettres et écritures ou même la Barre du jour, mais ces canaux ne nous offrent pas souvent l'espace suffisant ou approprié. Il faut créer par nousmêmes autre chose. Trouver un autre lieu.

Une autre scène. justement. Ce sera le vénérable collège Saint-Marie, rue de Bleury, en plein centre-ville montréalais. On y a là beaucoup d'ambitions nouvelles. Peut-être même devenir la deuxième université francophone de la métropole. On y recrute alors, non sans attention, de nouveaux effectifs enseignants - laïcs - au moment où la vocation jésuite s'effondre.

La maison que jouxte l'église du Gésù et son théâtre offre-t-elle à qui y pénètre les odeurs de réfectoire dont parle $\mathbf{R}$. Charbonneau dans sa Chro- 
nique de l'âge amer (1967, p. 29)? En fait, c'est le remugle typique du collège classique québécois qui vient aux narines, dans les boiseries craquantes et poussiéreuses des couloirs et escaliers. En ces lieux (installés en 1848) dont le ravalement hâtif ne masque pas une forte tradition, règne un vice-recteur laỉc, une autre tête à pipe et à lunettes, le paternel et libéral Paul-Émile Gingras. Plus haut, du côté des chambres où logent surtout quelques jésuites en demi-retraite de l'enseignement ou de la pastorale, se trouve Ernest Gagnon, l'enseignant déjà cité, l'essayiste novateur de l'Homme d'ici (1952 et 1963). L'analyste de l'intelligence «canadienne", le passionné de la symbolique dans l'art reste toujours disponible, recevant ses amis, même malade au lit, dans le petit capharnaüm de sa cellule. Il s'inquiète de tout, de sa collection d'art primitif africain dont des collègues, dit-il, veulent le délester, de son enseignement négligé, de l'avenir toujours. Son masque se creuse de plus en plus, dans une contre-épreuve blanche de ses chers objets primitifs: son oil noir, bulbeux, colle au verre épais de ses lunettes, ses joues blanchâtres rougissent par endroits dans le bleuté de sa barbe négligée. Une cigarette sartrienne pend à sa lippe amère.

C'est entre la chambre du moine relégué et celle du vice-recteur que navigue un jeune enseignant laïc de la maison, Robert Lahaise. Historien formé à Laval (par les Trudel et Lacourcière), il repasse par ces couloirs et escaliers qu'il a fréquentés étudiant, y retrouve les fantômes familiers de son père le poète Guy Delahaye et de tous les écrivains qui y ont étudié: Émile Nelligan, Victor Barbeau, Albert Laberge. Paul Morin. Saint-Denys Garneau, Robert Elie, Ringuet. Marcel Dubé, Pierre Perreault, Jacques Brault, André Brochu, André Vanasse... et que fréquentent maintenant des inconnus comme Yolande Villemaire et Claude Beausoleil.

Lahaise a un projet: publier les études et recherches des enseignants du Collège. Cela s’appellera "les Cahiers de Sainte-Marie». Pour les étudiants et les autres intéressés. Le premier sera titré Littérature canadienne, sous la responsabilité de Raymond Turcotte, professeur au département de français que dirigent Pierre Grenier et André Vanasse. S'y trouvent aussi Noël Audet, Renald Bérubé, René Dionne, Madeleine Greffard, Maximilien Laroche, Renée Legris et Jean-Guy Sabourin. J'y arriverai en juin 1966. Viendront bientôt André Belleau, Michel van Schendel et Hubert Aquin. Toutes personnes qui fonderont avec d'autres le département d'Études littéraires de l'UQAM. quand le vieux collège servira comme d'autres institutions montréalaises, de matrice à la nouvelle université du Québec.

\section{La mission de Littérature canadienne}

Le numéro paraît au printemps 1966, affichant en deuxième page de couverture une longue liste des divers responsables dont le "Conseiller" Ernest Gagnon, s.j.. Son nom ajouté à ceux des autres légitime de toute évidence le caractère "universitaire" ou sérieux d'une maison qui se donne avec cette première publication, une structure éditoriale. Robert Lahaise (directeur général) en annonce laconiquement la naissance et les objectifs, ceux déjà mentionnés plus haut, mais aussi l'éventuelle réimpression de "vieux 
textes canadiens». On trouve ensuite uñ "éditorial" de R. Turcotte, «Une démarche des premiers temps». À l'indifférence existant à l'égard de la littérature canadienne, il faut, dit-il, opposer le travail de véritables "missionnaires" et de "défricheurs»: brandir une foi à renverser les montagnes de pessimisme qu'on a toujours dressées contre l'existence même de notre littérature; assumer, selon le mot cité de Jean Simard, notre vocation de premiers intellectuels d'un peuple de bûcherons; ou encore (ce qui pour nous est plus juste), selon la formule de Jacques Brault, une tâche... écrasante, mais belle et nécessaire, puisque cette littérature, méconnue et méprisée est le firuit d'hommes (de poètes et de romanciers surtout) qui. dans des conditions pénibles, tentent de donner voix à un mutisme légendaire.

C"est dans cette perspective enthousiaste (trop? il y a sans doute à cette époque plus d'écrivains que de bûcherons professionnels) et modeste (des "cahiers" et non de savants ouvrages) que se dessine une nouvelle mission éditoriale et pédagogique. Personne ne prétend là révolutionner la critique littéraire, quoi qu'il apparaisse assez clairement aujourd'hui que cette initiative doive être incluse dans la progressive "nationalisation" du patrimoine littéraire, ou si l'on préfère la reconnaissance collective qui s'ensuit pendant les années soixante.

La première étude proposée (par R. Bérubé) prend les motifs fondateurs de la neige et de l'hiver. (dans le Temps des hommes) pour objet: Mon pal's. c'est l'hiver dira bientôt Gilles Vigneault. Vient ensuite l'analyse (de M. Greffard) d'Une saison dans la vie d'Emmanuel où s'affirme l'acteur écrivain (Jean-le-Maigre) contre une tradition carnavalisée. Puis celle (par R. Legris) de Prochain épisode où le pays impossible fait éclater l'espace narratif. Celle (par $R$. Turcotte) consacrée à deux romans "sociaux" des années cinquante. Au pied de la pente douce et Au milieu la montagne. Celle (par C. Racine) d'Ashini où l'indianité mythique, religieuse, propose en vain une rencontre transculturelle, universelle. Enfin celle (par moi) de la Petite poule d'eau où la réconciliation des différences canadiennes passe dans la rêverie d'une pastorale trans-ethnique.

Ainsi vus ces choix de textes et de thèmes sont moins qu'innocents: ils révèlent en bonne partie les enjeux de nos «sixties». Qui fera le détail des analyses verra bien tous les signes d'une quête identitaire bien connue, tout en ayant quelque difficulté à isoler une orientation carrément ethnocentrique. En revanche, ces analyses fortement thématiciennes ou, plus timidement. narratologiques, trahiront aisément leur pente sociologisante. Notre lecture est là portée sur le repérage du social et de sa référence, plutôt que sur un sens langagier, structuraliste ou esthétique. La mission néo-nationaliste ne se montre vraiment que chez $\mathrm{R}$. Turcotte pour qui les romans de Lemelin et Viau sont le s!mbole d'une nation jeune, en pleine montée... dont on a tout lieu d'espérer une maturité féconde (p. 47).

Dans cette canadianité des études (proclamée en couverture) circule aussi, on l'aura remarqué. l'indianité. Mais c'est l'indianité traditionnelle (ou poétique!), errante, celle qui ne laisse pas de trace, déterritorialisée jusque 
dans son aboutissement spirituel catholique ( "Mon Royaume n'est pas de ce monden). Trait qu'il faut rapprocher de la proposition finale de la Petite poule d'eau.

Même quand intervient ultimement l'américanité de $M$. Laroche ( $\mathrm{La}$ conscience américaine de la nouvelle poésie québécoise $)$ ), cette autre facette de la québécité montante n'est vue que comme une étape vers l'universalité: le national n'est pas exalté; c'est l'enracinement, l'authenticité qui sont raisonnés. Entre Jean Le Moyne (Convergences) qui désespère de notre capacité francophonique (parce que nous ne sommes pas en France) et Ernest Gagnon (I'Homme d'ici) qui souhaite un enracinement français et latin dans un pays nordique et anglo-saxon, le choix eșt vite fait en faveur de la dernière attitude. Conformément à notre tradition critique depuis Casgrain et Camille Roy. pour autant qu'on en reste surtout à la fidélité linguistique et culturelle.

Conclusion: porté par l'indéniable vecteur nationaliste du temps, ce premier "Cahier de Sainte-Marie" a participé à la quête identitaire québécoise sans folklorisme, sans parti-pris autre que l'élection d'un domaine d'études, le nôtre. L'entreprise avait ses limites, mais aussi ce mérite.

\section{Voix et images du pays}

Le deuxième cahier littéraire des Éditions de Sainte-Marie paraît en avril 1967. Sa couverture colorée parle autant que la parcheminée de Littérature canadienne. Sur ses bandes fragmentées de bleu et d'orangé sur fond blanc s'écrit d'abord en majuscules, à gauche pour sauter tout de suite à l'oil lecteur; LITTÉRATURE QUÉBÉCOISE. C'est le surtitre. On trouve plus bas, à droite: VOIX et IMAGES DU PAYS. La deuxième page de couverture annonce d'autres changements: Lahaise a remplacé Turcotte comme directeur des Cahiers et je suis le responsable du «Cahier de littérature". En troisième page de couverture, on signale que les Cahiers relèvent dorénavant du Secrétaire général et que si l'on peut se procurer deux autres cahiers déjà parus, il n'en va pas de même pour Littérature canadienne qui est épuisé...

S'il fallait sans nul doute pavoiser, mettre les couleurs officielles du bleu et du blanc (voir le drapeau du Québec avec son bleu de France et le redoublement de ses fleurs de lys), pourquoi l'orangé? S'agissait-il du rouge révolutionnaire décoloré par le jaune (papal! traître!) québécois? Plus banal ou curieux? je voyais dans ces couleurs celles d'une certaine peinture figurative traditionnelle comme chez Clarence Gagnon dans son "Charlevoix ». image d'ailleurs retenue (malheureusement rendue en noir et blanc) dans le numéro. C'était pendant ces années où nous recueillions avec ferveur ce qui restait de meubles anciens encore bon marché après la publication de l'ouvrage de Palardy. Nous achetions aussi ces fameuses catalognes qui allaient reservir au moment du "trip" fermier contre-culturel. Quoi qu'il en soit, toute cette couverture joue sur l'opposition France-Québec, sur la transformation de la francité par la québécité. Littérature "québécoise" répond à Études "françaises" (ce sera plus net en 1975 quand Voix et images sera sur-titré "d'études québécoises"). Et puis "Voix et images du pays» adapte(!) "Voix et images 
de France", nom d'une méthode alors existante de l'enseignement du français (langue seconde). (Comme la plupart des intellectuels québécois, je suis à cette époque "hainamouré" de France, militant de l'enseignement du français standard et de la littérature québécoise, fondant - en 1967 - l'Association des professeurs de français de Montréal, tout en dénonçant les manuels états-uniens mal traduits, avant d'aller en France y faire une thèse de doctorat en littérature française). C'était au temps de la québécité galopante, garrochée, rêvée.

Voix et images du pays, c'est à dire celles projetées par les écrivains du passé et du présent. Soit: le territoire imaginaire. fictionnel et poétique. Voilà pourquoi, dans ma présentation, je donne la parole à Gatien Lapointe et le premier espace à son Ode au Saint-Laurent (Montrez-moi cet homme de mon pa!s.. I Une image dè l'homme très jeune/plantant son corps dans l'espace et le temps).

Le mot clé, on le devine, reste le mot substitué: «pays». Mis en titre de la publication, il se répercutera plusieurs fois dans les titres des articles et circulera avec redondance dans le corps des analyses. C'est le territoire littéraire qui se placarde ainsi, mais aussi les petites patries de D. Potvin et Saint-Denys Garneau, le pays perdu de la Dalle-des-Morts (Savard). Et bien évidemment une lecture de la collectivité québécoise représentée, particulièrement chez. Bessette (le Libraire), Thériault (Agaguk) et Ferron (Contes du pays incertain). Il est indéniable que la vision d’ensemble des textes analysés et des analyses elles-mêmes est politique: relative à la vie d'une société organisée, à l'exercice d'un certain pouvoir. Mais le niveau est local, tribal, clérical, en un mot: traditionnel. On chercherait en vain une grande imagination de l'État, du Gouvernement. de l'économie ou de la vie politique même partisane. Et le tout est fait de l'essentielle ambiguïté littéraire... dont vivait peut-être notre société elle-même.

Comment s'étonner que les illustrations choisies soient: "La ferme» de Suzor-Côté, "Bois brûlé» de Gécin, "Cap-aux-Oies» de P. Faucher et «Charlevoix" de C. Gagnon? On est là fort peu "révolutionnaire». On y a de grandes tendresses pour le pays ailleurs contesté par certains d'entre nous. Où passent les analyses des récits "sociaux" de Parti pris ou "politiques" à la Godbout ou façon Aquin, dans lesquels se transcrit même l'actualité terroriste? Dans d'autres supports que ceux du Collège. Là où les risques du traitement à chaud sont plus faciles à assumer. même quand nous restons plutôt démunis côté munitions : où aurions-nous pris les moyens méthodologiques de véritables analyses "politiques "? Notre formation toujours en cours. demeure alors relativement traditionnelle : historicienne, thématicienne, avec une sensibilité sociologique ou linguistique (saussurienne) ou esthétique. Et si certains d'entre nous sont "socialistes», c'est d'un socialisme de papier, rebutés que nous sommes par le texte marxiste.

Dans ces conditions, il était plus simple de valoriser le méprisé, le déclassé, jusqu'au régionalisme à la Potvin, tout le patrimoine littéraire que 
l'institution marginalisait. Et puis nous pouvions toujours nous justifier théoriquement en nous situant dans la phase "pré-révolutionnaire" dont on parlait à la revue Parti pris. Pour.ma part. je voyais dans le Libraire. un texte précurseur des récits « révolutionnaires» de 1965-1966, un texte défricheur du silence, conquérant de la parole, contre la mainmise cléricale et "française" (normative) sur notre expression authentique. Je croyais ainsi prolonger la réflexion de J.-C. Falardeau sur notre roman. Ou même celle de Marcotte (Une littérature qui se fait), qui faisait lui aussi partie de notre mince bagage bibliographique. Nous étions en fait plus influencés par les auteurs de fictions que par les critiques ou les théoriciens. Nous chérissions particulièrement les poètes, comme Chamberland ou Giguère dont l'Áge de la parole pouvait nous «programmer".

C'est d'ailleurs ce mot "parole» (la construction personnelle du langage) qui investit et ordonne le numéro suivant du cahier littéraire: Voix et images du pays IJ. Renald Bérubé en est maintenant le maître d'œuvre. Il donnera à la collection des huit cahiers à venir son profil définitif. respectant le format originel et son esprit, mais préparant par étapes la revue quı paraîtra à compter de 1975.

Bérubé reprend d'abord, en la prolongeant, la problématique de Littérature canadienne et de Voix et images I, mais en donnant plus d'espace que jamais aux "créateurs", notre essentielle référence. Après Loranger (Un cri qui vient de loin, dans Littérature canadienne, après la lettre autographe de Saint-Denys Garneau (Voix et images du pays I), voici les signatures de Brault. P.-M. Lapointe et Aquin qui s'ajoutent à celle du fidèle A. Major (deux textes déjà dans Voix et images du pays I).. Il s'agit bien du "Devoir de parole", ainsi que le titre Bérubé dans sa présentation, en reprenant le registre missionnaire de Turcotte.

En 1970, l'Université du Québec à Montréal naissante absorbe beaucoup des effectifs du Sainte-Marie. R. Lahaise continue tout naturellement ses "Cahiers" aux Presses de l'Université du Québec qui s'y trouvent, par la même occasion, lancées. La troisième livraison de Voix et images du pays paraît. Bérubé affirme sa volonté de continuité, pour que les écrivains d'ici $v$ soient entendus et reconnus... par des lecteurs toujours plus nombreux... II ajoute: Nous voulons que Voix et Images du Pays véhicule la culture québécoise - l'homme d'ici et l'originalité de sa perception du monde - et participe au mouvement de clémocratisation de celle-ci. La continuité se marque jusque dans les signatures récurrentes: Audet. Laroche, Lavoie. Aquin, Lapointe et Major sont encore au rendez-vous avec Languirand et Châtillon.

Les numéros IV et $V$ surviennent selon la même formule, quoique s'affaiblisse la profession de foi au pays, dans les avant-propos comme dans les articles. L'un de ces derniers enfonce sans doute un clou tout à fait magistral. II s'agit de "Notes sur le thème du pays" par Gilles Marcotte lui-même. On sait que cette thématique ne va pas de soi chez lui (voir son essai bref dans Canadian Literature). Mais grâce à lui et à d'autres collaborateurs (L. Mailhot, J. Pelletier, B. Dupriez) les notes infra-paginales augmentent 
substantiellement. La revue devient plus «informée», "universitaire». D'ailleurs elle a gagné en volume. Le numéro 111 (1970) comptait déjà plus de trois cents pages...La collection annuelle a dès lors son créneau, sa légitimité, son autorité. Petit changement dans la structure éditoriale: le nom de R. Lahaise a disparu depuis le no IV (197I). On saura qu'il a quitté les P. U.Q. dirigées maintenant par Thomas Déri, pour aller fonder les "Cahiers du Québec» chez Hurtubise-HMH...

Le caractère scientifique de la collection s'affiche encore davantage en 1973 quand elle se dote d'un comité de rédaction de style "université du Québec", puisqu'il sera en lui-même un "réseau ", interuniversitaire et pancanadien avec, autour de Bérubé, J. Bonenfant (Sherbrooke). A. Brochu (U. de Montréal), R. Dionne (Ottawa), G. Lapointe (le poète enseigne maintenant à l'UQ à Trois-Rivières), A. Leblanc (Laval). P. -A. Arcand (Moncton), G. Bessette (Queen's), J.-M. Duciaume (Alberta), R. Giguère (Vancouver $\mathrm{UBC}$ ) et $\mathrm{J}$. Pelletier (Rimouski). La publication a même son protocole de rédaction.

Dans l" "avant-présentation" de ce numéro VI, A. Brochu (au nom du nouveau comité de rédaction?) écrit que le temps du nationalisme lyrique est passé. qu'il faut construire notre identité culturelle qui est une facente de notre identité politique. Ou encore: promouvoir et approfondir l'idée d'une appartenance culturelle à ce pal's, encore incertain, qu'est le Quéhec. Par quels moyens? Sans doute ceux de cette publication restructurée que présente ensuite R. Bérubé: aux études et inédits s'ajoutent maintenant des «lectures d'œuvres" (ćtudes plus brèves) et des "Notes ou contre-notes" sur tout le culturel: politique, histoire, cinéma, sport... Pour la première fois, Bérubé parle d'une revue interuniversitaire, même si c'est d'abord une position politique qui est avancée (avec Brochu). Voix et images du pays prendra-t-elle la relève de Parti pris? Politisée, sera-t-elle la revue interuniversitaire du culturel? Dans le sillage de ces "sciences de la culture" qu'essaie de mettre sur pied un groupe de professeurs de l'UQAM? Le projet paraît plus fantasmé que réfléchi.

Cela est manifeste quand on passe au numéro suivant (VII) paru la même année 1973. L'avant-propos de Bérubé dit tout dans son titre: "Cohérence et continuité ou fatigue et piétinement". Dans ce temps de l'aprèsoctobre 1970 alors que le nationalisme terroriste a été écrasé par Ottawa, le thème de la continuité, du "pays" à définir ou approfondir est lourd à porter. Bérubé dit à la première ligne de son topo: la lecture des terres qui composent ce numéro... a quelque chose de passionnant (stimulant) et d'agaçant (frustrant? déprimant?") (șic) tout à la fois. C'est que la quête identitaire évoque pour lui le temps répétitif de Trente arpents. Le style embarrassé, mimétiquement itératif, de son texte crayonne lourdement sur le motif du piétinement (dans la continuité).

La présentation du numéro qui suit (VIII. 1974) s'intitule: "avant-propos continu continuel ». Est-il possible de marquer davantage la lassitude? L'ouverture du numéro qui suit ( $1 \mathrm{X}^{\mathrm{e}}$ et dernier) dit tout simplement: "Avant- 
propos (suite)" ... Fatigué de publier l'analyse du piétinement. Bérubé dit aussi celle d'écrire ces textes censés inciter le lecteur à lire les articles qui suivent... D'où sa pratique mimétique du «stream of consciousness» et finalement (en IX) du style énumératif qu'il veut administratif, pour dire:

A Voix et images du pays, c'est connu (certitude ou souhait?) (sic) l'aventure littéraire n'est jamais dissociée de l'aventure d'un pa!ls qui se fait. se défait, se refair. Nous y crolons...

Pathétique cette insistance du discoureur qui a d’autant plus besoin d'afficher le crédo initial qu'il en est venu à amputer le nom même de sa publication: à plusieurs reprises, dans son texte, le "pays" disparaît. À la dernière ligne, il n'est question que de Voix et images. L'expression familière, écourtée, s’imprime déjà.

\section{Voix et images}

Quand, en 1974. T. Déri me demande de prendre la succession, je lui propose une revue, rattachée à un département d'Études littéraires, ayant une périodicité fixe (trois fois par an pour éviter les pavés de plusieurs centaines de pages). des abonnés et un format vraiment universitaire. i.e. dont les contributions fassent avancer la connaissance sur le sujet abordé. des bibliographies, des comptes rendus de recherche etc. Quel en sera le nom? Consultation faite dans l'entourage, on tient beaucoup au titre disponible. Ne s'agit-il d'ailleurs pas de continuer Voix et images du pays? A. Belleau insiste pour que je retienne le nom intégralement. Pour la continuité. Toujours. D'où peut donc venir pareil impératif? Avons-nous au Québec été à ce point inconstants?

La revue s'appellera donc Voix et images, ne serait-ce que pour démontrer la vérité hémonique du refrain de Maria Chapdelaine repris dans Menaud maître-draveur et Il n'y a pas de pays sans grand-père: Au pal's de Québec, rien ne doit changer. Mais on surtitrera: "études québécoises" plutôt que "Littérature.. " pour bien montrer que la parole est maintenant aux analystes plutôt qu'aux producteurs... Et puis pourquoi pas "voix" et "images": ces termes ont un usage codifié en critique et théorie littéraires?

Cette promotion de la continuité d'un esprit n'allait pas de soi en 19741975 quand il m'a fallu aller défendre une demande de subvention auprès d'un jury ministériel québécois. Le président en était G.-A. Vachon, alors directeur d'Études françaises. Flanqué d'un fonctionnaire. je fus requis de justifier la pertinence de Voix et images nouvelle manière à partir d'une évaluation faite du Voix et images du pays ancien style. Pour le jury, il ne s'agissait pas d'une revue universitaire. laissait entendre clairement le président (qui ne se sentait nullement en conflit d'intérêt. même s'il était lui aussi demandeur pour sa revue)... Études françaises devait suffire. C'était l'époque où la plus récente des universités avait aussi la plus mauvaise réputation.

Laissant à d’autres le soin du parcours qui reste à faire pour compléter la revue de cette revue de 1975, je conclus rapidement. 
D'où vient Voix et images? D'abord de la lecture fascinée de notre patrimoine littéraire au moment où lui vient à lui comme à toute notre société une conscience vive de ses moyens et de sa force imaginaires. En ce sens, Voix et images n'a pas été au départ une revue très critique. Elle était plutôt en elle-même un hommage. L'écho des fameuses voix hémoniques, comme elles passaient dans les écrits mêmes des écrivains du jour.

Elle vient aussi du support intelligent de deux structures scolaires montréalaises. l'une en pleine expansion. l'autre en train de donner naissance à une troisième. Elle vient en particulier des travaux souvent méconnus d'Albert Le Grand et d'Ernest Gagnon qui ont davantage enseigné que publié. Elle vient de l'acharnement de $R$. Lahaise a créer de nouveaux lieux éditoriaux. Elle vient du dévouement de $R$. Bérubé avant qu’il n’aille défricher ses terres rimouskoises.

Elle vient de jeunes enseignants et promoteurs de la québécité, dont je fus. En passant. 